\title{
Breast and prostate cancer: familial associations
}

\author{
Kari Hemminki, Asta Försti and Bowang Chen
}

In a recent Opinion article by Gail Risbridger and colleagues, Breast and prostate cancer: more similar than different (Nature Rev. Cancer 10, 205-212 (2010)), the extensive biological similarities of the hormone-dependant pathways of breast and prostate cancers were discussed ${ }^{1}$. They also pointed out that mutation of $B R C A 2$ predisposes to both breast and prostate cancer, and that mutation carriers with prostate cancer have a poorer survival rate than patients with prostate cancer who do not have BRCA2 mutations ${ }^{2}$. However, whether the biological similarities would lead to a familial aggregation of these cancers was not discussed. Familial associations between breast and prostate cancer would imply a shared genetic susceptibility that might go beyond mutations in BRCA2. The recently characterized low-penetrance genes for these cancers provide few clues about a possible shared susceptibility, with the exception of the 8q24 locus. However, the associated single nucleotide polymorphisms found at this locus are not concordant ${ }^{3-6}$.

We have used the nationwide Swedish Family Cancer Database, the world's largest data set of its kind ${ }^{7}$, to study shared familial clustering of various cancers, including breast cancer with prostate cancer ${ }^{8}$. This study identified 10,553 sons ( 0 to 70 years old) and 107,518 fathers (unlimited age) with prostate cancer, among a total of 170,000 cancer patients in the offspring generation and more than 800,000 cancer patients in the parental generation. We calculated familial standardized incidence ratios (SIRs) and confidence intervals (CIs) for prostate cancers and other cancers in family members ${ }^{8,9}$. Separate proband groups were used: affected parents only, affected siblings only, and affected parents and siblings, to suggest genetic modes of inheritance and level of penetrance ${ }^{10}$. The methods of calculation have been described.

For breast cancer, we found that the SIRs were 1.64 , when the mother was diagnosed with breast cancer, 1.91 when the sister was diagnosed and 3.83 when both mother and sister were diagnosed. In discordant analysis when probands were diagnosed with prostate cancer, the SIRs for breast cancer were 1.11, 0.96 and 1.62. For prostate cancer, each of the concordant risks was higher, particularly for brothers (3.40), and fathers and brothers (6.71); the corresponding SIRs for prostate cancer in breast cancer families were 1.19 when the mother was diagnosed with breast cancer, 1.07 when the sister was diagnosed and 1.55 when both mother and sister were diagnosed. All these discordant SIRs were significant at a 5\% level (TABLE 1), except those among siblings; many were significant even at a $1 \%$ level. When prostate cancer was diagnosed before the age of 60 years in sons of a mother with breast cancer the SIR was $1.47(n=41,95 \%$ CI 1.17-1.82) ${ }^{8}$.

Considering that BRCA2 mutations account for only a small proportion of familial breast cancers in Sweden it is unlikely that the common parent-offspring aggregation of breast and prostate cancers would be explained by these mutations, except in the high-risk families of parent and sibling probands ${ }^{11}$. However, the median diagnostic age of breast cancer was 54 years in families with both father and brother diagnosed with prostate cancer, and this was higher than that found in BRCA2 mutation carriers $(<50 \text { years })^{12}$. Therefore, these data suggest that only a small proportion of the prostate-breast aggregation in families was explained by BRCA2 mutation and that new shared susceptibility genes are likely to exist.
Kari Hemminki, Asta Försti and Bowang Chen are at the Division of Molecular Genetic Epidemiology, German Cancer Research Center (DKFZ). Im Neuenheimer Feld 580, 69120 Heidelberg, Germany.

Kari Hemminki and Asta Förstiare also at the Center for Primary Care Research, Lund University. Malmö 20502, Sweden.

Kari Hemminki is also at the Center for Family and Community Medicine, Karolinska Institute Huddinge 14183, Sweden.

Correspondence to K.H. e-mail: K.Hemminki@dkfz.de doi: $10.1038 /$ nrc2795-c1

\section{Acknowledgements}

The Family Cancer Database was created by linking registers maintained at Statistics Sweden and the Swedish Cancer Registry. Supported by Deutsche Krebshilfe and the Swedish Council for Working Life and Social Research.

\section{Competing Interests \\ The authors declare no competing financial interests.}

Risbridger, G. P., Davis, I. D., Birrell, S. N., Tilley, W. D. Breast and prostate cancer: more similar than different. Nature Rev. Cancer 10, 205-212 (2010). Tryggvadottir, L. et al. Prostate cancer progression and survival in BRCA2 mutation carriers. J. Natl Cancer Inst. 99, 929-935 (2007).

3. Easton, D. F. et al. Genome-wide association study identifies novel breast cancer susceptibility loci. Nature 447, 1087-1093 (2007).

4. Eeles, R. A. et al. Multiple newly identified loci associated with prostate cancer susceptibility. Nature Genet. 40, 316-321 (2008)

5. Gudmundsson, J. et al. Common sequence variants on 2 p 15 and Xp 11.22 confer susceptibility to prostate cancer. Nature Genet. 40, 281-283 (2008).

6. Thomas, G. et al. Multiple loci identified in a genomewide association study of prostate cancer. Nature Genet. 40, 310-315 (2008)

7. Hemminki, K., Li, X., Plna, K., Granström C \& Vaittinen, P. The nation-wide Swedish Family-Cancer Database: updated structure and familial rates. Acta Oncol. 40, 772-777 (2001)

8. Hemminki, K. and Chen, B. Familial association of prostate cancer with other cancers in the Swedish-FamilyCancer Database. Prostate 65,188-194 (2005).

9. Hemminki, K., Vaittinen, P., Dong, C. \& Easton, D. Sibling risks in cancer: clues to recessive or $\mathrm{X}$-linked genes? Br. J. Cancer 84, 388-391 (2001).

10. Hemminki, K., Sundquist, J. \& Lorenzo Bermejo, J. Familial risks for cancer as the basis for evidence-based clinical referral and counseling. The Oncologist 13, 239-247 (2008)

11. Lorenzo Bermejo, J. \& Hemminki, K. A populationbased assessment of the clustering of breast cancer in families eligible for testing of BRCA1 and BRCA2 mutations. Ann. Oncol. 16, 322-329 (2005).

12. Chen, $\mathrm{S}$. et al. Characterization of BRCA1 and BRCA2 mutations in a large United States sample. J. Clin. Oncol. 24, 863-871 (2006).

\section{Table 1 | Familial risk of breast and prostate cancer

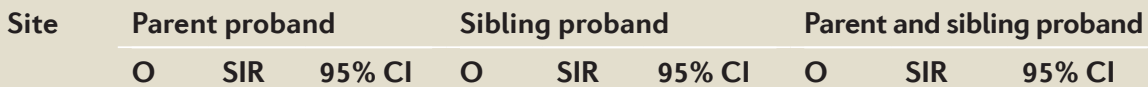

Familial risk for concordant prostate and breast cancer

$\begin{array}{llllllllll}\text { Breast }^{\star} & 3656 & 1.64^{\ddagger \S} & 1.59-1.70 & 1855 & 1.91^{\ddagger \S} & 1.79-2.04 & 248 & 3.83^{\ddagger \S} & 3.38-4.33\end{array}$

Risk of breast cancer in families with patients with prostate cancer

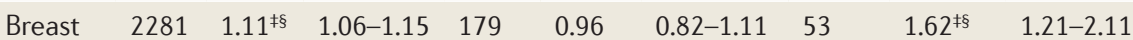

Risk of prostate cancer in families with patients with breast cancer

\begin{tabular}{|c|}
\hline ostate \\
\hline
\end{tabular}

$\mathrm{Cl}$, confidence interval; $\mathrm{O}$, number of observed cases; $\mathrm{SIR}$, standardized incidence ratio.*All data were taken from REF. 8, except the Breast* row, which originates from REF. $10 .{ }^{\ddagger} 99 \% \mathrm{CI}$ does not include 1.00 . $\S 95 \% \mathrm{Cl}$ does not include 1.00 . 